\title{
INTERVIEW
}

For reprint orders, please contact: reprints@futuremedicine.com

\section{An interview with Giuseppe Argenziano: an insight into the field of dermoscopy}

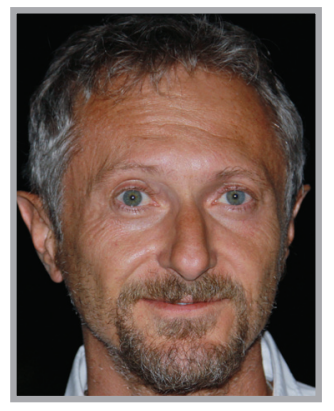

Giuseppe Argenziano* speaks to Sebastian DennisBeron, Commissioning Editor: Giuseppe Argenziano is Full Professor and Head of the Dermatology Unit at the University of Campania, Naples, Italy. His main research field is dermato-oncology. He is an author of numerous scientific articles and books concerning dermoscopy, a new technique improving the clinicians detection of benign and malignant skin tumors. As a coordinator of a Skin Cancer Unit, he has established a successful tertiary, multidisciplinary, referral center particularly devoted to the diagnosis and management of patients with skin tumors. Over the past 20 years he has supervised over 100 foreign students and 40 residents in dermatology, established scientific collaborations with more than 200 colleagues from more than 30 nations, and organized more than 50 national and international scientific activities, courses and conferences (such as the Consensus Net Meeting on Dermoscopy and the First Congress of the International Dermoscopy Society). He is co-founder and past president of the International Dermoscopy Society; project leader for the development of a high diagnostic technology oncologic center at the Arcispedale Santa Maria Nuova IRCCS in Reggio Emilia; faculty member of the Master of Science in Dermoscopy and Preventive Dermato-oncology and has undertaken a short course in dermoscopy, two e-learning courses by the Medical University of Graz and by Cardiff University, respectively; and member of the Editorial Board of the Journal of the American Academy of Dermatology. Professor Argenziano has authored more than 400 full scientific articles and produced landmark primary publications and books in the field of dermoscopy. Over the past 20 years, he has been invited as speaker and/or chairman in more than 500 national and international conferences in the field of dermatology. His combined publications have received a sum total of more than 8000 citations with an h-index value of 46.

Accepted for publication: 5 August 2016; Published online: 3 March 2017

\section{Q What inspired you to develop a career in dermatology?}

I have a very strong family influence; my father is a dermatologist and my grandfather was a dermatologist. So in some way you could say I was attracted by my family's history in the field. My work in melanoma specifically, began quite early in my career; I was in contact with patients with melanoma and other skin cancers as my medical degree thesis was on these particular issues, and this is why I was very early attracted by this topic.

\section{Q Did you have any colleagues that} were strong influences in your career? There are two main people that influenced my professional development. One is, first, my father who taught me a lot and second was P Soyer (University of Queensland,

\section{KEYWORDS}

- dermoscopy • melanoma • skin cancer 
Australia). Soyer, a colleague in dermatology, who worked in Graz, Austria for many years, is now chairman of the Dermatology department in Brisbane, Australia. He was basically my mentor from a scientific perspective because he introduced me to the science of dermoscopy and melanoma. We spent many hours studying together.

\section{Q What would you consider your biggest professional achievement to date?}

There are two main experiences I could mention: those related to my patient care and second, those related to my teaching. In terms of my teaching, where I count my main achievements, I can recollect an experience a few years ago where I taught a course on recognizing melanoma. Following the course, a colleague went home and she was able to recognize a particularly difficult melanoma to diagnose. This colleague then explained that because of this course she was able to recognize this lesion as melanoma where she was not able to do so before hand. This is essentially what I try to achieve every day. Not only do I work with patients and try to do the best that I can for them, but also crucially, I aim to improve the quality of my colleagues' professional work and in turn be able to potentially influence the diagnosis of many more patients, on top of the ones I see face to face.

\section{Q You recently departed as President of the} International Dermoscopy Society. During your time there, how did you see the field of dermoscopy grow?

Dermoscopy has grown a lot in the last 15-20 years; I was acting president of the International Dermoscopy Society for 6 years and during those 6 years, my main goals were to improve the spread of the technique worldwide. When I started as president we had 2000-3000 members, which has now increased to upward of 8000 . Within the society, we published a lot of papers in the field of dermoscopy and we promoted a lot of events, such as conferences and congresses to increase access and understanding of the field. As a result, today in many countries, dermoscopy is a basic tool that all dermatologists use in their practice. In the last 10 years, we have been able to bring thousand dermatologists into the field of dermoscopy which in my view is one of our greatest advancements

There are two real important landmarks in the last few years, which I would say were two main consensus meetings, one consensus published in
2000 and another which was recently published in 2016. Consensus meetings are guidelines which all dermatologists refer to; in the consensus of 2000 , we were able to establish a basic methodology on how to use the dermatoscope, breaking down the methods and algorithms used to reach a particular diagnosis.

The main goal of the 2016 consensus paper was to bring together the analytic terminology and the metaphoric terminology to develop a more detailed description of the various criteria we can see through the dermatoscope. As a result, we were also able to get rid of redundant terminologies which were published in the last few years and, therefore, have a more synergistic group of criteria.

\section{Q Your research focuses on the use of developing more accurate methods for the early recognition of melanoma. How important is the use of dermoscopy not only in melanoma diagnostics, but also in its management?}

The main goal of dermoscopy is its use as a diagnostic tool, but it can also be used for the management of melanoma. We are able to use dermoscopy for monitoring a lot of skin cancers, which are treated with nonsurgical methods. For example, we use it a lot when we use topical treatments to treat basal cell carcinoma, since this is not a surgical procedure. Using the technique, you can verify that the use of the cream was able to completely remove the basal cell carcinoma. This is one example in which we can use dermoscopy in the field of management.

This is also true in all dermatology patients, not only skin cancer patients. Dermoscopy can be used as a diagnostic technique for a variety of dermatological issues outside skin cancer, such as psoriasis and eczema.

\section{Q There is a lot of discussion regarding} the use of teledermatology in clinics. How would you describe this emerging field \& how important is its impact on patient care? Teledermatology is a very interesting technique that has developed a lot in the last 20 years, with many papers being published in that time. However, it is only able to help if we are dealing with a country where there are very few dermatologists. For example, in Italy there are 4000 dermatologists. As a result, no patient will ask for a consultation via teledermatology, as there is simply no need for it. 
In countries like Australia or in the UK, where there are fewer dermatologists, it is extremely important to add this technique to increase patient access. Dermatology is a perfect field for teleconsultation, because in the vast majority of cases you are able to diagnose and manage the patient with just a picture.

Paradoxically, teledermatology is much simpler in the general dermatology field, than in the skin cancer field. In skin cancer, teledermatology does not really suffice, as although you have the morphology of the lesion in an image, you still need to grasp how rest the of the patient's lesions look, understand the patient skin type and have an idea of their patient history. But in general dermatology, teledermatology is extremely useful; $5 \%$ of my daily work involves consulting with my patients via WhatsApp ${ }^{\circledR}$. Many patients send pictures of their lesions and you can easily solve very benign problems, usually in a minute, saving a lot of time.

There is the potential for teledermatology to have a strong impact if used correctly. Aside from diagnosis, you can monitor the patient treatment, for example, through a very common way of exchanging images between the patient and consulting dermatologist, to ensure the treatment is working.

\section{Q As the field of melanoma rapidly evolves,} how important is it for clinicians to collaborate while managing the disease, from diagnosis through to treatment?

Melanoma and skin cancers in general, are multidisciplinary diseases. As a dermatologist, you act as the center and you work with various other disciplines in order to treat and manage the patient.

As dermatologists we have to understand the latest developments in melanoma therapy, since we are connecting the patients from one specialist to the other. We need to be aware of the latest possibilities and guidelines available. We must collaborate with other specialists, like the pathologists, the radiologists and the oncologists, to determine the needs of the patient and work around them.

The workflow is quite straightforward; if you see a lesion suspected to be melanoma, you need the pathologists to get the final diagnosis. The patient is then sent to the surgeon for the sentinel node biopsy, and then if there is something suspicious discovered by the dermatological follow-up, you have to decide the plan of action, be it surgical or medical, together with the surgeons and oncologists.
Q What barriers stand in our way in terms of expanding the field of dermoscopy?

The known barriers are still the economic ones, with clinicians claiming that they do not have a dermatoscope in their office as it is too expensive. This should not be an issue as dermatoscopes are now approximately US $\$ 400$. The biggest barrier, I believe, is the lack of training in the field, though nowadays in many countries, there are teaching programs on going where dermatologists are being trained in the technique.

I would not say that there are really important barriers in terms of moving forward and spreading the technique and in my view, in another 10 years, dermoscopy will be a standard in all dermatological consultations. It will be like ophthalmologists not using their tools to look into the eyes - it is simply not possible.

\section{Q What direction do you see the field taking} in the future?

There is still much to do. You can see from the number of publications; every year there is an increasing number of papers being published in dermoscopy, which means the field is still expanding.

Though basic aspects of the field have already been established, there are of course some areas that still need to be improved, such as recognizing facial melanomas and amelanotic melanomas which are still very difficult lesions to diagnose.

There will be more and more papers published in the field of general dermatology, as each of the dermatological conditions has its own dermoscopic counterparts. It means, in my view, that the future of dermoscopy will focus additionally on the nontumoral field in general dermatology, other than skin cancers.

\section{Disclaimer}

The opinions expressed in this interview are those of the interviewee and do not necessarily reflect the views of Future Medicine Ltd.

Financial \& competing interests disclosure

$G$ Argenziano has no relevant affiliations or financial involvement with any organization or entity with a financial interest in or financial conflict with the subject matter or materials discussed in the manuscript. This includes employment, consultancies, honoraria, stock ownership or options, expert testimony, grants or patents received or pending, or royalties.

No writing assistance was utilized in the production of this manuscript. 